Noname manuscript No.

(will be inserted by the editor)

\title{
Shockwaves in spillways with the particle finite
}

\section{element method.}

\author{
Fernando Salazar • Javier San-Mauro • \\ Miguel Ángel Celigueta · Eugenio Oñate
}

Received: date / Accepted: date

\author{
Abstract Changes in direction and cross-section in supercritical hydraulic \\ channels generate shockwaves which result in increase in flow depth with re- \\ F. Salazar · J. San-Mauro · M. Á. Celigueta · E. Oñate \\ Centre International de Mètodes Numèrics en Enginyeria (CIMNE). Universitat Politècnica \\ de Catalunya (UPC), Campus Norte UPC, Gran Capitán s/n. 08034. Barcelona, Spain \\ Tel.: +34-934-071-495 \\ F. Salazar \\ E-mail: fsalazar@cimne.upc.edu \\ J. San Mauro \\ E-mail: jsanmauro@cimne.upc.edu \\ M.Á. Celigueta \\ E-mail: maceli@cimne.upc.edu \\ E. Oñate \\ E-mail: onate@cimne.upc.edu
}


gard to that for uniform regime. These disturbances are propagated downstream and need to be considered in the design of the chute walls. In dam spillways, where flow rates are often high, this phenomenon can have significant implications for the cost and complexity of the solution. It has been traditionally analysed by means of reduced-scale experimental tests, as it has a clear three-dimensional character and therefore cannot be approached with two-dimensional numerical models. In this work, the ability of the particle finite element method (PFEM) to reproduce this phenomenon is analysed. PFEM has been successfully applied in previous works to problems involving high irregularities in free-surface. First, simple test cases available in the technical bibliography were selected to be reproduced with PFEM. Subsequently, the method was applied in two spillways of real dams. The results show that PFEM is capable of capturing the shockwave fronts generated both in the contractions and in the expansions that occur behind the spillway piers. This suggests that the method may be useful as a complement to laboratory test campaigns for the design and hydraulic analysis of dam spillways with complex geometries.

Keywords Particle finite element method · Spillways $\cdot$ Shockwaves

\section{Introduction}

Shockwaves are frequently generated in dam spillways by uniform flow disturbances due to the presence of piers, curves or changes in the cross-section [34]. As a consequence, local maxima in flow depth are produced whose magnitude 
can be much greater than the incoming uniform depth. This has important practical implications in the dimensioning of the chute walls, which must be designed with greater height to adequately convey the flow, with the obvious consequences in terms of magnitude and cost.

The design of the spillway must tend to the constant cross-section and slope with the aim of approaching uniform flow and thus avoiding this phenomenon. However, these geometric particularities are frequently unavoidable due to the topographical and/or geological characteristics of the dam and spillway site.

The hydrodynamic study of spillways has historically been carried out using small-scale experimental tests. The computational methods available during the period of greatest intensity in dam design and construction did not allow these complex problems to be addressed, since they are not treatable with two-dimensional models [22]. However, the numerical tools currently available can be used for studying this type of 3-D problems with sufficient detail and with an assumable computational cost.

Numerical models are often used for the design and analysis of spillways in combination with physical model tests [31], [30]. This allows the numerical model to be validated for the geometry considered, so that the versatility of the latter can subsequently be used to extend the catalogue of situations analysed in alternatives studies. In addition, numerical models allow a more detailed analysis of aspects that are difficult to measure in the laboratory, such as stream lines or pressure in areas at risk of cavitation [32], [36]. 
Numerical models have been primarily used to evaluate the discharge capacity of spillways [36], [26] and to optimize the approach conditions [31], thanks to the ease with which flow patterns can be analysed. The numerical schemes employed include eulerian [2], [26], and lagrangian approaches such as smoothed particle hydrodynamics (SPH) [29].

However, the analysis of shockwaves is much less frequent. Some examples with quasi-horizontal bottom have been addressed with depth-integrated approaches, which offered accurate results (e.g. [37], [33]). However, spillway chutes often feature steep slopes, which limits the applicability of $2 \mathrm{D}$ simplifications. The particle finite element method (PFEM) is a lagrangian approach for the numerical modelling of fluid dynamics problems among many other applications [1]. The fact that the PFEM does not need a background mesh, and thus the user does not need to foresee what parts of the domain will be occupied by the fluid in later stages of the simulation, makes it especially appropriate to consider phenomena with strong changes in the free surface both in time and space [9], [8].

The method has been employed to face a variety of problems in different fields of engineering, such as seakeeping [12], landslide-generated waves [25], [27], industrial forming processes [18], ground excavation [3], fluid-structure interaction [13], among others [15],[14], [7]. PFEM has also been previously applied to solving 3-dimensional free-surface flows, particularly in hydraulic structures [10]. It has also been employed to estimate air flow demand in bottom outlets [28], [20]. 
This paper explores the possibilities of using PFEM in full-scale hydrodynamic analysis of spillways with geometric irregularities that generate shockwaves. Firstly, it has been applied to reference cases available in the classical literature including curves and channel contractions. Then, two examples of application to real dam spillways are presented, with different characteristics: the first features a mild slope and an abrupt convergence, reducing the useful width of the channel by more than $50 \%$ in a length similar to the initial width. In the second case, the convergence is smoother, although it allows for a detailed analysis of the negative waves generated behind the piers.

The article is completed with a brief reminder of the basis of the PFEM implementation used in this work and a discussion on the possibilities and limitations, as well as ideas for future work.

\section{Numerical model}

The PFEM is a documented method in the literature [19], well suited for continua with large deformations and separation. In the PFEM, the domain is modelled using an Updated Lagrangian formulation [38]. That is, all variables are assumed to be known in the current configuration at time $t$. The new set of variables in the domain are sought for in the next or updated configuration at time $t+\Delta t$. The finite element method (FEM) is used to solve the equations of continuum mechanics. Hence a mesh discretising the domain must be generated in order to solve the governing equations in the standard FEM fashion. When the mesh gets too distorted due to the large deformations it must be 
rebuilt to avoid inverted elements. The re-meshing step can be used to improve the mesh quality as well. The PFEM is therefore a compound method consisting in a combination of the Finite Element Method, an Updated Lagrangian formulation and a re-meshing algorithm.

The equations to be solved for a single phase fluid (like water) are the Navier-Stokes equations for incompressible fluids:

Momentum conservation

$$
\rho \frac{D u_{i}}{D t}=-\frac{\partial}{\partial x_{i}} p+\mu \frac{\partial}{\partial x_{j}}\left(\frac{\partial u_{i}}{\partial x_{j}}\right)+\rho f_{i}
$$

for $i, j=x, y, z$

Mass conservation

$$
\frac{\partial u_{i}}{\partial x_{i}}=0
$$

for $i=x, y, z$

with

$$
u=\bar{u}
$$

for the wall nodes (considered as fixed fluid nodes) and

$$
p=0
$$

for the free surface fluid nodes.

In the equations above, the unknowns are $p$ (pressure) and $u$ (velocity). $\rho$ and $\mu$ are the fluid density and dynamic viscosity, respectively, $u_{i}$ are the velocities along the $i$ th global (cartesian) axis, $f_{i}$ are the volumetric accelerations 
(only gravity acceleration is used in this work), and $\bar{u}$ is the prescribed velocity. Note that fixing the three components of the velocity to zero is equivalent to assuming a no-slip boundary condition. This type of boundary condition was used in every fluid-wall interphase.

The Lagrangian formulation implies that the nodes move with the fluid velocity. In particular, the nodes located at the free surface follow the evolution of the boundary in a very accurate, natural way.

According with the PFEM technique [19], equations 1 and 2 are discretised with a standard FEM mesh and then solved by means of an implicit, Fractional Step method. The elements used are simplex, 4-noded tetrahedra, with linear interpolation of both the pressure and the velocity. When the finite elements get very distorted, the mesh is re-generated, but the nodes and their information are conserved. Adaptive mesh refinement techniques can be used to improve the solution in zones where large deformations of the fluid occur.

The details of the algorithm and our implementation was described in previous publications [13], [16], [17]. In this paper, only the basic steps of the algorithm are succinctly described, together with some enhancements specifically implemented for the present application.

\subsection{Basic steps of the PFEM}

In the PFEM, the mesh nodes in the fluid and solid domains are treated as particles that contain all the information of geometry, material and mechanical properties of the underlying subdomains. 
A typical solution with the PFEM involves the following steps.

1. The starting point at each time step is the cloud of points $C$ in the fluid and the walls. The walls domain simply contains fluid nodes which have an externally imposed velocity and can be moved as a rigid body. For instance, ${ }^{n} C$ denotes the cloud at time $t={ }^{n} t$ (Fig. 1).

2. The domain is discretised with a finite element mesh ${ }^{n} M$ using the particles as the mesh nodes. We use an efficient mesh generation scheme based on the Delaunay tesselation [9].

3. The free surface is detected by means of the Alpha Shape Method [6], a geometrical criterion which removes the elements coming from the Delaunay Tesselation that are not considered as part of the fluid domain. The Alpha Shape Method compares the diameter of the circumsphere of each element with a reference, maximum allowed diameter. Bigger circumspheres correspond to either big elements (connecting distant nodes) or flat, distorted elements which can only be present in areas where the density of nodes drops considerably (in areas with a homogeneous density of nodes the Delaunay Tesselation generates high quality tetrahedra). Note that the free surface is not the border between two fluids, but the external boundary of the domain considered fluid.

4. The Lagrangian equations of motion for the overall continuum are solved using the standard FEM. The state variables in the next (updated) configuration for ${ }^{n} t+\Delta t$ are computed: velocities, pressure, strain rate and viscous stresses. 
5. The mesh nodes are moved to a new position ${ }^{n+1} C$ where $n+1$ denotes the time ${ }^{n} t+\Delta t$, in terms of the time increment size.

6. Go back to step 1 and repeat the solution for the next time step to obtain a new ${ }^{n+1} C$.

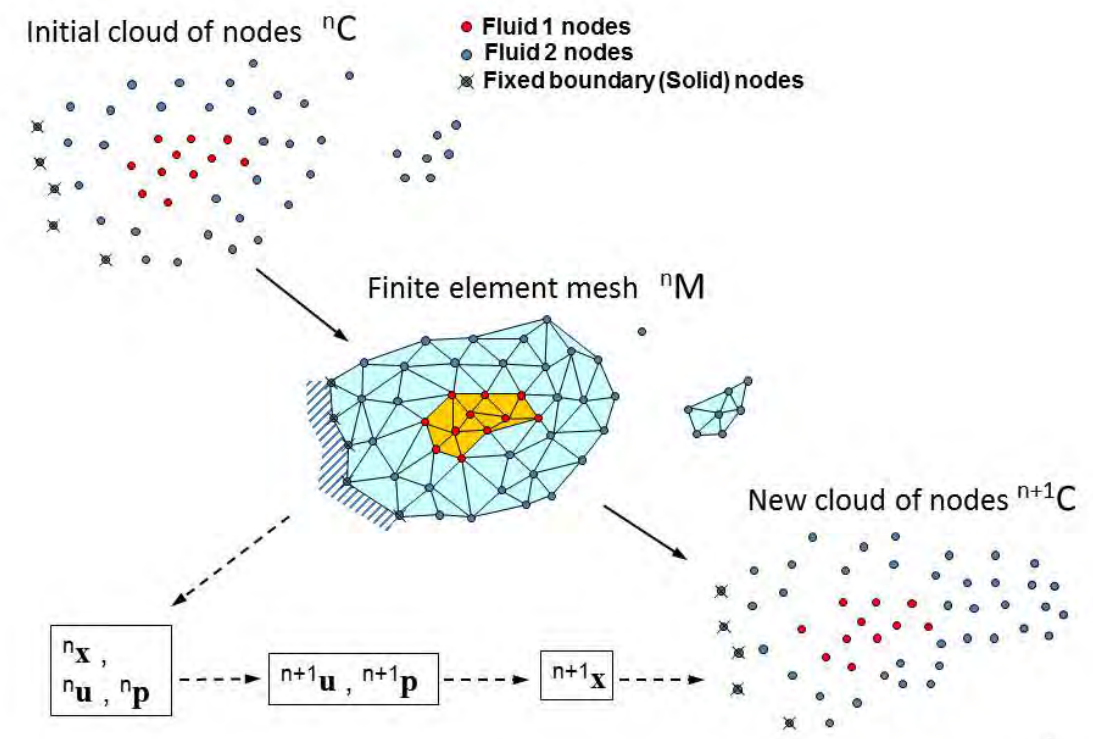

Fig. 1: Sequence of steps to update a "cloud" of particles (nodes) representing a domain containing two fluids and a solid boundary from ${ }^{n} t$ to ${ }^{n+1} t$.

2.2 Inlets

Fluid inlets are necessary when modelling spillways or channels as an upstream condition. Being the PFEM a fully Lagrangian method, the inlet condition 
cannot just be modeled as a Dirichlet condition for the velocities because the nodes at the inlet surface get transported with the velocity field and move out of the surface where they were originally located. Thus, in the PFEM used for this work, inlets are conceived as successive layers of nodes that are inserted in the domain at a given location (usually a surface). Figure 2 depicts a scheme of the process in 2D. Every new layer of nodes must be considered exactly in the same way as a solid wall, with a Dirichlet velocity condition (step 2). For several time steps (step 3 ) the boundary condition is maintained until a certain distance from the inlet is reached and a new layer of nodes is inserted (step 4). This distance must be similar to the size of the elements. The old nodes are freed and the new ones are fixed to the same velocity as before. At this stage, both layers get connected by elements that are created automatically by the re-meshing process. After this, the newest nodes move with the imposed velocity (step 5) until they are replaced by a new layer of nodes in the same way it was done before (step 6).

\section{Benchmark cases}

Some classical cases have been collected to verify the ability of the PFEM to model irregularities in supercritical flow. They are described in this section. Only the essential information is included, since the experiments are well documented in the reference articles, which are conveniently cited. 
1

2

3
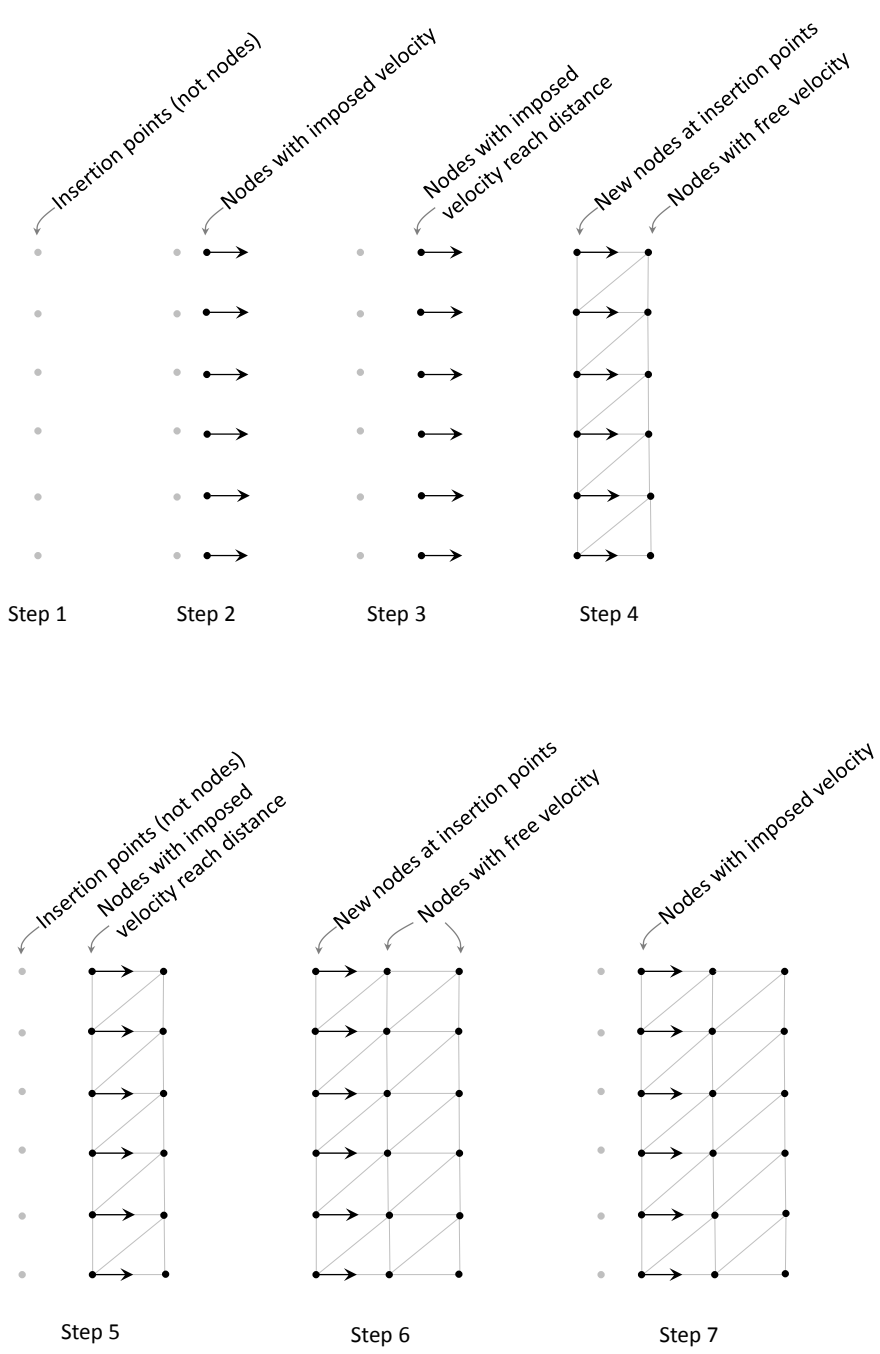

Fig. 2: Schematic process of node injection at an inlet

3.1 Chute bend

The change in the flow direction generated in a circular bend in supercritical regime results in two shockwaves: a positive one producing increase in flow 
depth and a negative one with depth below uniform. Figure 3 shows a scheme of the phenomenon.

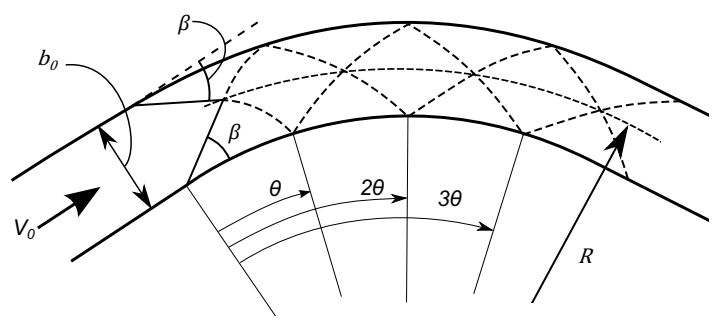

Fig. 3: Shockwave pattern in a rectangular channel bend with supercritical flow. Adapted from [5]

The angle $\beta$ formed by both wave fronts with the initial flow direction depends on the Froude number of the incoming flow $\left(F_{0}\right)$ and can be approximated by the expression ([5], p.452)

$$
\sin (\beta)=\frac{1}{F_{0}}
$$

In turn, $\theta$ can be computed as:

$$
\tan \theta=\frac{\frac{b_{0}}{R}}{\left(1+\frac{b_{0}}{2 R}\right) \tan \beta_{0}}
$$

Where $b_{0}$ stands for the channel width and $R$ is the average radius of curvature. The maximum flow depth $h_{\max }$ can be estimated as:

$$
h_{\max }=h_{0} F_{0}^{2} \sin ^{2}\left(\frac{1}{F_{0}}+0.5 \theta\right)
$$


Table 1: Bend in rectangular channel. Main features.

\begin{tabular}{llll}
\hline & & Case B1 & Case B2 \\
& $b_{0}$ & $0.5 \mathrm{~m}$ & $0.5 \mathrm{~m}$ \\
Channel width & $R$ & $3.25 \mathrm{~m}$ & $4.25 \mathrm{~m}$ \\
Average curve radius & $h_{0}$ & $0.05 \mathrm{~m}$ & $0.05 \mathrm{~m}$ \\
Flow depth at inlet & $F_{0}$ & 4 & 4 \\
Froude number at inlet & $v_{0}$ & $2.18 \mathrm{~m} / \mathrm{s}$ & $2.8 \mathrm{~m} / \mathrm{s}$ \\
Inflow velocity & & $1 \mathrm{~cm}$ & $1 \mathrm{~cm}$ \\
Mesh size & & $2.1910^{6}$ & $2.1210^{6}$ \\
Number of elements & & $0.186 \mathrm{~m}$ & $0.153 \mathrm{~m}$ \\
Theoretical maximum flow depth (Eq.7 ) & $h_{\max }$ & $0.15 .0^{\circ}$ & $23.3^{\circ}$ \\
Theoretical location of maximum (Eq. 6) & $\theta$ & $29.0^{\circ}$ & \\
\hline
\end{tabular}

Two configurations have been modelled with the PFEM with parameters shown in Table 1, which also includes the theoretical values of the $h_{\max }$ and $\theta$ obtained with equations 6 and 7 .

Figure 4 shows the result of the numerical simulation, with both the location and the value of the first theoretical maximum flow depth. The exact location of $h_{\max }$ is not easy to identify in the numerical results, since the free surface at the outer wall is close to horizontal along a certain area. Nonetheless, the theoretical approximation falls within that area. The numerical result for $h_{\max }$ is $0.180 \mathrm{~m}$ and $0.159 \mathrm{~m}$ for $R=3.25 \mathrm{~m}$ and $R=4.25 \mathrm{~m}$ respectively, i.e. the deviation with respect to the theoretical maxima is $3.2 \%$ and $3.9 \%$.

The results for the second and third maxima are less accurate (Table 2 and 3 ), especially for $2 \cdot \theta$. This might be due to numerical diffusion, but also to the relatively high curvature, which can result in deviation from the theoretical 
Table 2: Chute bend (Case B1). Flow depth maxima. Theoretical vs numerical results

\begin{tabular}{lllll}
\hline Location (angle) & Flow depth $(\mathrm{m})$ & & \\
\hline & Theoretical & & Numerical & \\
& Inner wall & Outer wall & Inner wall & Outer wall \\
& 0 & 0.186 & 0 & 0.180 \\
$2 \cdot \theta$ & 0.186 & 0 & 0.042 & 0.094 \\
$3 \cdot \theta$ & 0 & 0.186 & 0 & 0.144 \\
\hline
\end{tabular}

Table 3: Chute bend (Case B2). Flow depth maxima. Theoretical vs numerical results

\begin{tabular}{lllll}
\hline Location (angle) & Flow depth $(\mathrm{m})$ & & & \\
\hline & Theoretical & & Numerical & \\
& Inner wall & Outer wall & Inner wall & Outer wall \\
$\theta$ & 0.002 & 0.153 & 0 & 0.159 \\
$2 \cdot \theta$ & 0.153 & 0.002 & 0.06 & 0.09 \\
$3 \cdot \theta$ & 0.002 & 0.153 & 0 & 0.122 \\
\hline
\end{tabular}

values [33]. Another possible source of deviation from the theoretical value is wave breaking [23], which indeed is observed in Figure 4.

\subsection{Contraction}

An abrupt contraction in a hydraulic channel in supercritical regime generates shockwaves resulting in increase in flow depth propagating downstream [34]. 

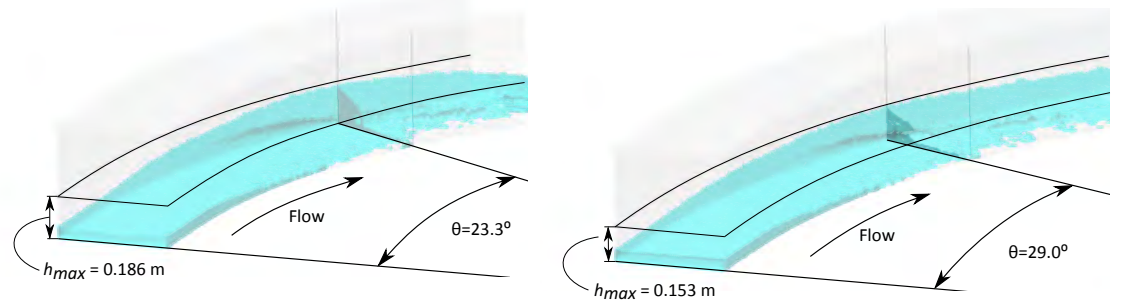

Fig. 4: Chute bend. The expected empirical values for $h_{\max }$ and $\theta$ are overimposed on the numerical results. Left: Case B1; $R=3.25 m$. Right: Case B2; $R=4.25 m$
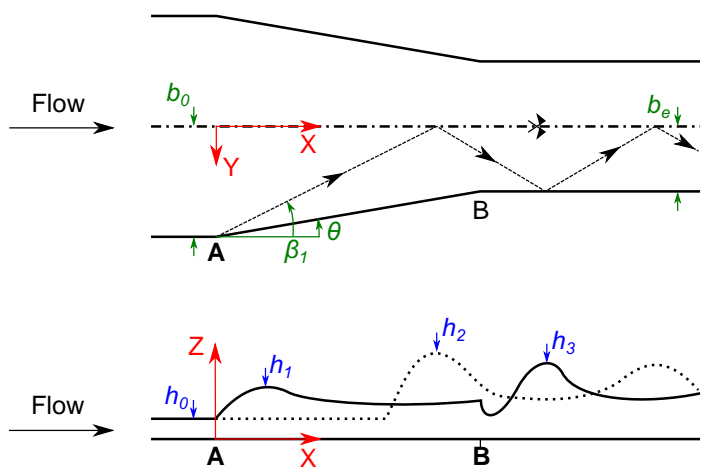

Fig. 5: Shock waves in a contraction. Local maxima in the axis (dotted line) and in the walls (solid line). Adapted from [24]

Reinauer and Hager [24] described the shape and location of the shockwaves both along the walls and the axis, as depicted in Figure 5.

The angle of the shock wave front can be estimated with the expression [21]:

$$
\tan \theta=\frac{\tan \beta_{1}\left(\sqrt{1+8 F_{0}^{2} \sin ^{2} \beta_{1}}-3\right)}{2 \tan \beta_{1} 1+8 F_{0}^{2} \sin ^{2} \beta_{1}-1}
$$


Where: $\theta$ is the angle formed by the chute wall and the direction of the incoming flow, $\beta_{0}$ is the shockwave angle and $F_{0}$ is the Froude number before the contraction.

Reinauer and Hager [24] proposed expressions to calculate the maximum flow depth on the walls and axis, as well as the location of the first maximum:

$$
\begin{gathered}
X_{1}=(2+0.126 \alpha) \theta+(1.4-0.015 \alpha) \\
h_{1}=h_{0}\left(1+\frac{1}{\sqrt{2}} S_{0}\right)^{2} \\
h_{2}=h_{0}\left(1+\sqrt{2} S_{0}\right)^{2} \\
h_{3}=h_{0}\left(w^{-1}+1.86 S_{0}^{0.5}-0.2 \alpha^{0.6}\right) \\
w=\frac{b_{e}}{b_{0}} \\
S_{0}=\theta F_{0}
\end{gathered}
$$

Where $X_{1}$ is the location of $h_{1}, h_{1 . .3}$ is the value of the three first maxima, alternatively located on the wall and the axis. $\alpha$ is the channel slope. $\theta$ is the angle formed by the wall with the initial flow direction. $h_{0}, F_{0}$ and $b_{0}$ are the flow depth, the Froude number and the channel width before the contraction, respectively. $b_{e}$ is the channel width downstream of the contraction. 
Table 4: Parameters for the contractions analysed

\begin{tabular}{llll}
\hline & & Case C1 & Case C2 \\
Channel slope & $\theta(\mathrm{rad})$ & 0.186 & 0.169 \\
Angle of the chute wall & $R$ & $3.25 \mathrm{~m}$ & $4.25 \mathrm{~m}$ \\
Channel width at inlet/outlet & $b_{0}(\mathrm{~m})$ & $0.5 / 0.3$ & $0.5 / 0.15$ \\
Flow depth at inlet & $h_{0}$ & $0.05 \mathrm{~m}$ & $0.05 \mathrm{~m}$ \\
Froude number at inlet & $F_{0}$ & 6 & 6 \\
Inflow velocity & $v_{0}$ & $4.2 \mathrm{~m} / \mathrm{s}$ & $4.2 \mathrm{~m} / \mathrm{s}$ \\
Mesh size & & $1.2 \mathrm{~cm}$ & $1.2 \mathrm{~cm}$ \\
Number of elements & & $1.2910^{6}$ & $1.1110^{6}$ \\
\hline
\end{tabular}

Two of the settings available in the literature have been selected to be modelled with the PFEM to assess its capability to reproduce shockwaves in these configurations, with different conditions. The parameters defining both tests are shown in Table 4.

The maximum mesh size in both cases was set to $12 \mathrm{~mm}$. This value ensured having at least 3 elements in depth in the whole domain. Also, only half of the original geometry was considered, taking advantage of the symmetry of the design.

\subsubsection{Contraction case C1}

Figure 6 shows a general view of the numerical result, where the flow depth maxima can be identified. They are alternatively located on the wall and axis, as expected. The theoretical values of $X_{1}, h_{1}, h_{2}$ and $h_{3}$ have been computed with Equations 9, 10, 11 and 12. A direct comparison with the numerical results 


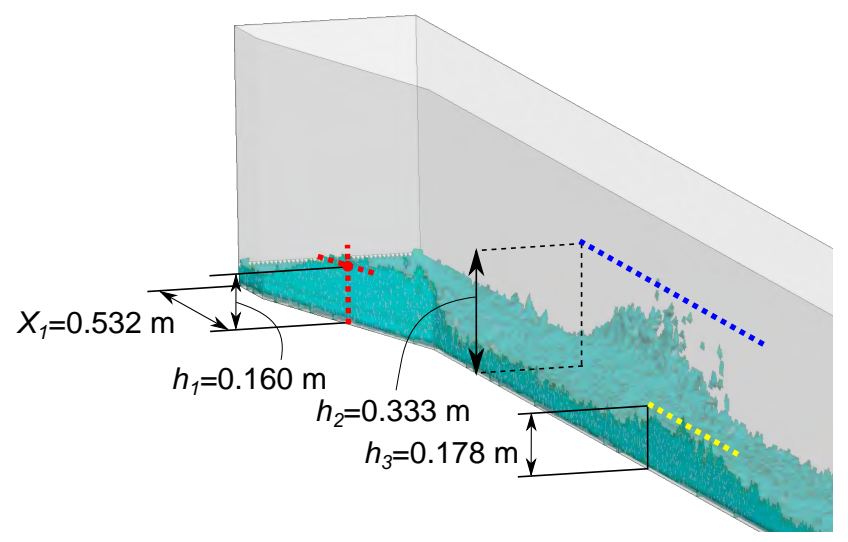

Fig. 6: Contraction case C1. The expected empirical values for $X_{1}, h_{1}, h_{2}$ and $h_{3}$ were placed over a view of the numerical result.

cannot be made, since the intrinsic properties of PFEM result in irregular shape of the free surface, with some elements separating from the main fluid body. Therefore, planes - horizontal for $h_{1}, h_{2}$ y $h_{3}$ and vertical for $X_{1}$ - have been drawn and superimposed on the numerical results for comparison. It can be seen that all results lie within the correspondent location.

The plan view of the numerical result (Figure 7) allows approximating the resulting angle $\beta_{1}$ formed by the shockwave front, which in this case presents some curvature. The estimated direction $\left(18^{\circ}\right)$ is $5 \%$ lower than the empirical estimate computed with Equation $8\left(19.2^{\circ}\right)$.

\subsubsection{Contraction case C2}

In this case, the results of the PFEM were compared to the experimental measurements published by Reinauer [21]. For that purpose, the profiles of the 


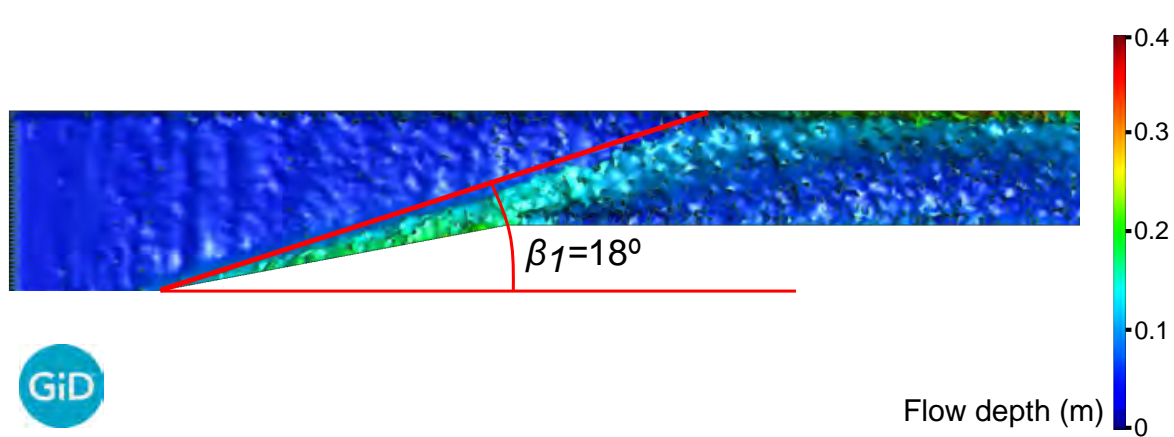

Fig. 7: Contraction C1. Numerical results (plan view). The shockwave front features a small curvature. Its direction can be estimated as forming $18^{\circ}$ with the main flow direction. This is $5 \%$ lower than the empirical estimate computed with Equation $8\left(19.2^{\circ}\right)$.

free surface along the chute wall and axis were extracted and superimposed over the experimental ones in Figure 8. The results coincide qualitatively in terms of the alternation of maxima between the axis and the wall, including the local minimum in the wall immediately downstream of the contraction. The main discrepancy is observed in the second maximum on the axis, which moves downstream in the numerical model.

As in the previous case, the angle of the shockwave front has been approximated and compared to the empirical value given by Equation 8. The result is shown in Figure 9.

The difference in $\beta_{1}$ is coherent with the location of the flow depth maxima in the numerical model, slightly downstream as compared to the empirical estimate (Figure 8). 

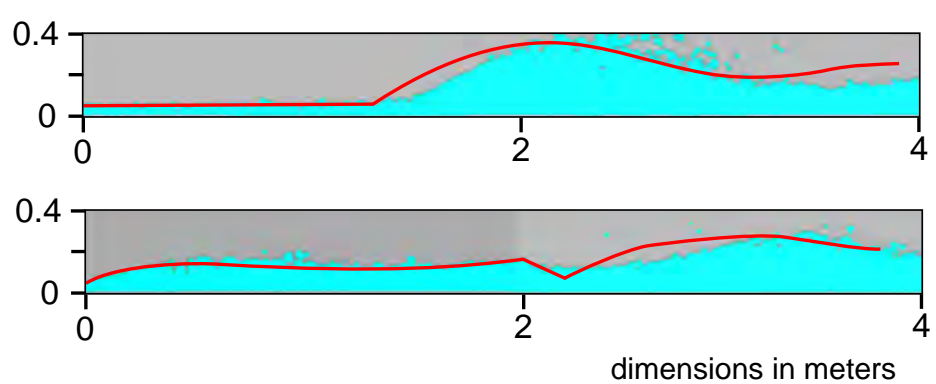

Fig. 8: Contraction case C2. Side view of the numerical results as compared with the flow depth measured in the experiment (red lines) [21]. Side view. Top: Chute wall. Bottom: Channel axis

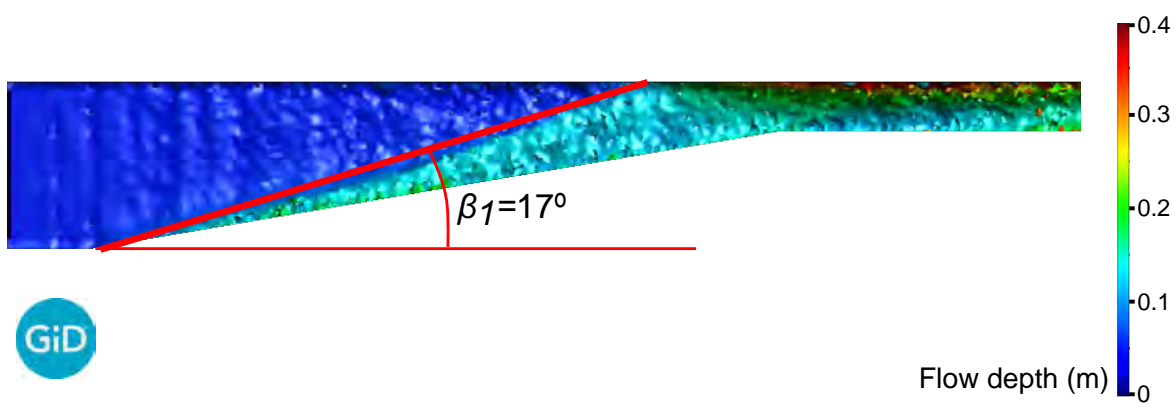

Fig. 9: Contraction case C2. Numerical results (plan view). The direction of the shockwave front can be estimated as forming $17^{\circ}$ with the main flow direction. As in the previous case, the empirical estimate given by Equation $8\left(18.2^{\circ}\right)$ is $5 \%$ higher.

\section{Real scale tests}

In this section, two cases are presented in which the PFEM is applied to analyse the hydraulic behaviour of spillways with different degree of convergence. Both share geometric features resulting in the formation of shockwaves. 
4.1 Case study \#1

\subsubsection{Model description}

The first spillway analysed includes a chute with a mild slope (11.1\%). It features four spans $13 \mathrm{~m}$ wide regulated by gates supported by three piers: the central pier is $3.5 \mathrm{~m}$ wide and has a rectangular profile at the downstream end, and the other two are $2 \mathrm{~m}$ wide, with downstream hydrodynamic profile and shorter length. The total width of the initial section is $59.5 \mathrm{~m}$ and after a transition along $55 \mathrm{~m}$ it joins the discharge channel, which has a constant useful width of $21 \mathrm{~m}$. In this case, the side channels feature sloping bottom to direct the flow towards the axis. In addition, they are slightly elevated with respect to the central channel, which in turn is aligned with that of the downstream chute. Given that the focus of this study is on the shockwaves generated by the change in cross-section, the energy dissipation structure was not analysed. Figure 10 shows the geometry and the main dimensions of the model.

A discharge rate of $1,800 \mathrm{~m}^{3} / \mathrm{s}$ has been considered. This value corresponds to one of the experimental tests for which data are available (the actual flow in the laboratory was obtained by applying hydraulic similarity to the aforementioned $1,800 \mathrm{~m}^{3} / \mathrm{s}$ ). The gates remain fully open while evacuating this flow, therefore the weir operates in free flow regime. This situation has been reproduced in the numerical model by applying an inlet boundary condition corresponding to the critical regime (flow depth $=4.98 \mathrm{~m}$; inflow velocity 


\subsubsection{Expected behaviour}

In addition to the convergence of the whole section, which goes from 59 to $21 \mathrm{~m}$ as mentioned above, the central channel also has a convergence so that its useful width goes from $26 \mathrm{~m}$ at the inlet (two bays) to $21 \mathrm{~m}$ of the chute. As there is also a step between the bottom of this area and that of the outer spans, additional shock waves are expected to occur. Both convergences are indicated in Figure 11. 

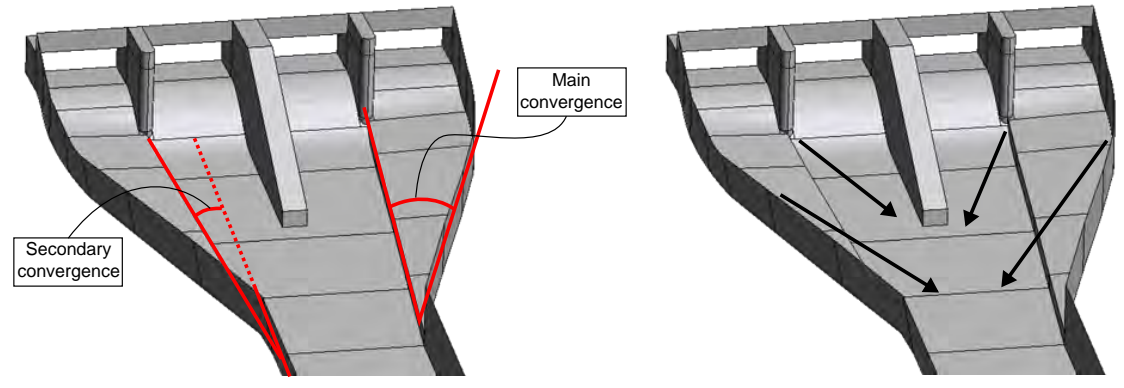

Fig. 11: Left: Convergence of the chute in Case study \#1. Right: Expected shockwaves

It is well known that contractions of this type with supercritical flows generate shockwaves propagating downstream, similar to those described in Section 3.2. The angle $\beta_{1}$ formed by the wave front can be obtained with Equation 8. According to this theoretical formulation, two pairs of wave fronts should appear, corresponding to the two width changes shown in Figure 11, which will propagate downstream.

\subsubsection{Numerical results}

The numerical modelling performed with PFEM has been capable to reproduce the formation of shockwaves observed in the physical model and described by Reinauer and Hager [24], both in the walls and in the spillway axis, due to the confluence of the shock waves (Figure 12).

The complex geometry of this spillway prevents local maxima from being estimated using the formulas mentioned above. As an example, $h_{2}$ in this case results from the combination of the increase in flow depth produced by the 


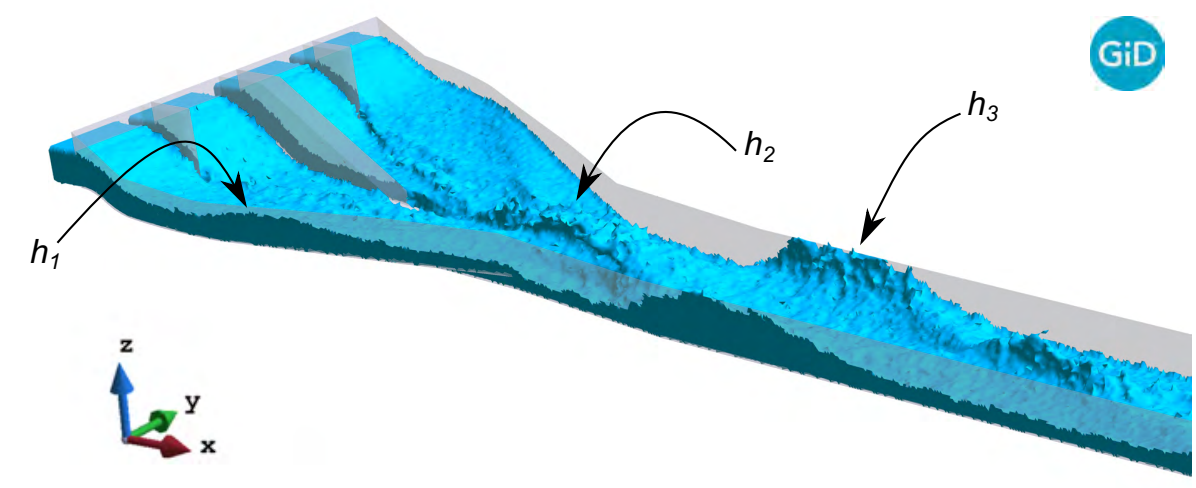

Fig. 12: Local maxima in the chute alternates between the walls and the axis.

convergence and that due to the expansion behind the central pier, known as roostertail [34], [35].

The numerical results have been compared to those obtained in the laboratory. Figures 13 and 14 include different views of the experiments and the corresponding numerical results. In the latter, the magnitude of the velocity component perpendicular (along the $Y$ axis in the reference system used) to the main flow direction ( $X$ direction) is plotted for better visualisation of the shockwaves. This and other variables, which require complex instrumentation to be measured in laboratory, can be easily analysed in the numerical models.

The results presented for this case study show PFEM's ability to qualitatively capture the phenomenon. For a quantitative evaluation, they have been compared to the theoretical values of the angle formed by the wave front, and to the flow depth measured in the laboratory with the considered flow rate. 

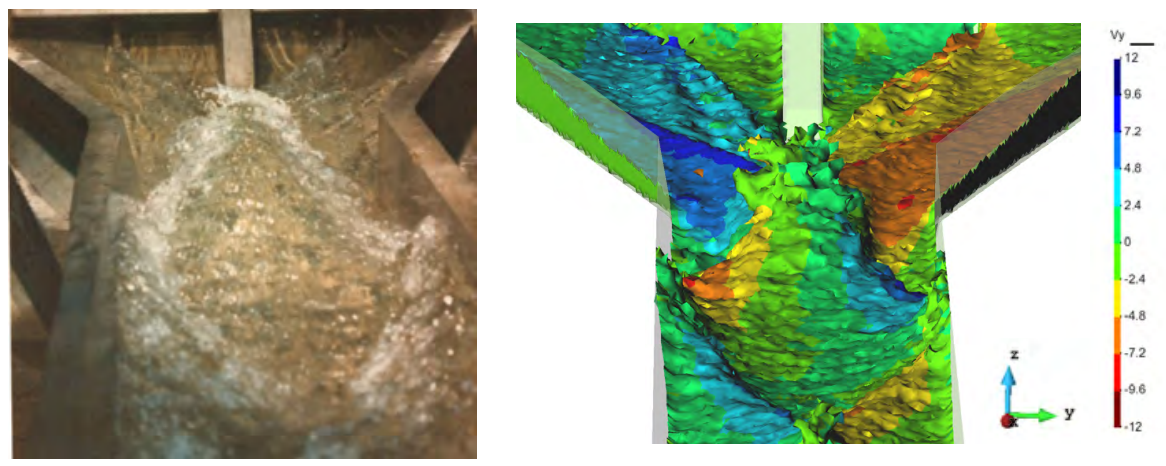

Fig. 14: Comparison between numerical and experimental results for Case Study \#1. View from downstream.

The theoretical direction of the wave front $\beta_{1}$ can be obtained with Equation 8 by entering the value of the incoming Froude number and the angle formed with the main direction of the channel. With the features of this case study, a $\beta_{1}$ value of $27.2^{\circ}$ is obtained for the secondary convergence. In Fig- 


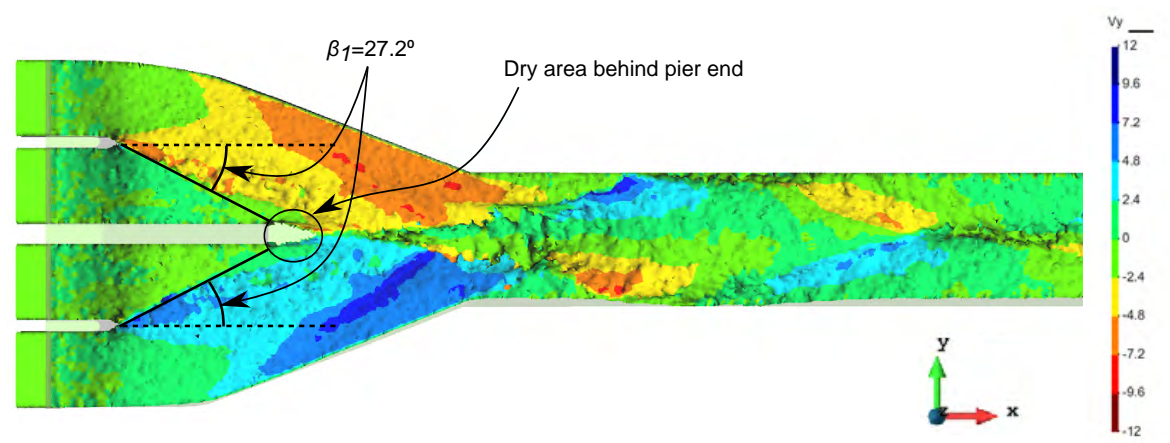

Fig. 15: Comparison between the empirical estimate for the shockwave front angle $\beta_{1}$ and the numerical results. The magnitude of the velocity component perpendicular to the main flow direction is plotted for better visualisation of the shockwave location.

ure 15 this direction has been superimposed on a detailed plan view of the numerical results.

Finally, the flow depth along the right chute wall has been compared to that registered in the laboratory. Figure 16 shows that the first two maxima are coincident in magnitude, although they are located slightly downstream in the numerical model. This behaviour is similar to that observed in section 3.2 .2 .

\subsection{Case study \#2}

The second real spillway modelled corresponds to a double curvature arch dam, with a height of $102 \mathrm{~m}$. The spillway is located on the left abutment, with 3 gate-controlled bays of $6.0 \mathrm{~m}$ separated by 2.0 -m-wide piers. The chute 
Fig. 16: Depth along the right chute wall. Comparison between the experimental results (red line) and the numerical model.

Table 5: Boundary conditions and mesh properties for Case study \#2

\begin{tabular}{lllll}
\hline Flow $\left(\mathrm{m}^{3} / \mathrm{s}\right)$ & Flow depth at inlet $(\mathrm{m})$ & Inflow velocity $(\mathrm{m} / \mathrm{s})$ & Mesh size $(\mathrm{m})$ & \# Elements \\
160 & 2 & 4.44 & 0.25 & $1.4810^{6}$ \\
450 & 4 & 6.25 & 0.3 & $1.4110^{6}$ \\
\hline
\end{tabular}

walls feature a mild convergence, with a total channel width changing from $22.0 \mathrm{~m}$ to $12.0 \mathrm{~m}$ at the entrance of the sky jump, after $90 \mathrm{~m}$ with a $45 \%$ slope.

The spillway has been reported to perform satisfactorily, as can be expected from such a gentle convergence. Nonetheless, piers typically generate local maxima termed roostertails, which were the main goal of this case to be modelled with PFEM. Two scenarios were considered, with discharge flow of 160 and $450 \mathrm{~m}^{3} / \mathrm{s}$. The upstream boundary condition was set to match with the critical flow under the gates, assuming free flow (Table 5).

Since no experimental results are available for this specific geometry, we compared the numerical results with empirical expressions to estimate the magnitude of the maximum flow depth behind the piers. Reinauer and Hager 
[22] described their location and shape for channels with horizontal bottom and Froude number above 5. For piers with rectangular end, they described a dry area limited by the flow from both sides of the pier which then collide and generate a local maximum. The waves propagate downstream and form a second local maximum once they reach the chute wall or another wave. These features can be observed in the numerical model.

The PFEM results show the roostertails behind the piers (Figure 17). As expected, the magnitude of the local maxima increased with higher flow rate. Figure 18 includes a close-up view.

The results show that the mild convergence in the chute width does not generate relevant shock waves.

The magnitude of the local flow depth maxima can be compared to the result of experimental formulas. The above mentioned study of Reinauer and Hager has been recently extended by Xue et al. [35] with additional experimental data including sloping bottom $i$. These authors propose the following expression to compute the maximum flow depth $h_{m}$ for piers with elliptical end of semi-major axis $a$ and $h_{0} / b \leq 1.5$, where $h_{0}$ is the flow depth at the tail part of the pier and $b$ is the pier width:

$$
h_{m} / h_{0}=2.5\left(h_{0} / b\right)^{0.5} \exp \left(-0.74 \cdot a / \cdot h_{0}-1.11 \cdot i\right)
$$

This expression cannot be directly applied to this case study, since the geometry of the pier end is not elliptical. Therefore, we verified that our numerical results are intermediate between those obtained for rectangular and 
1

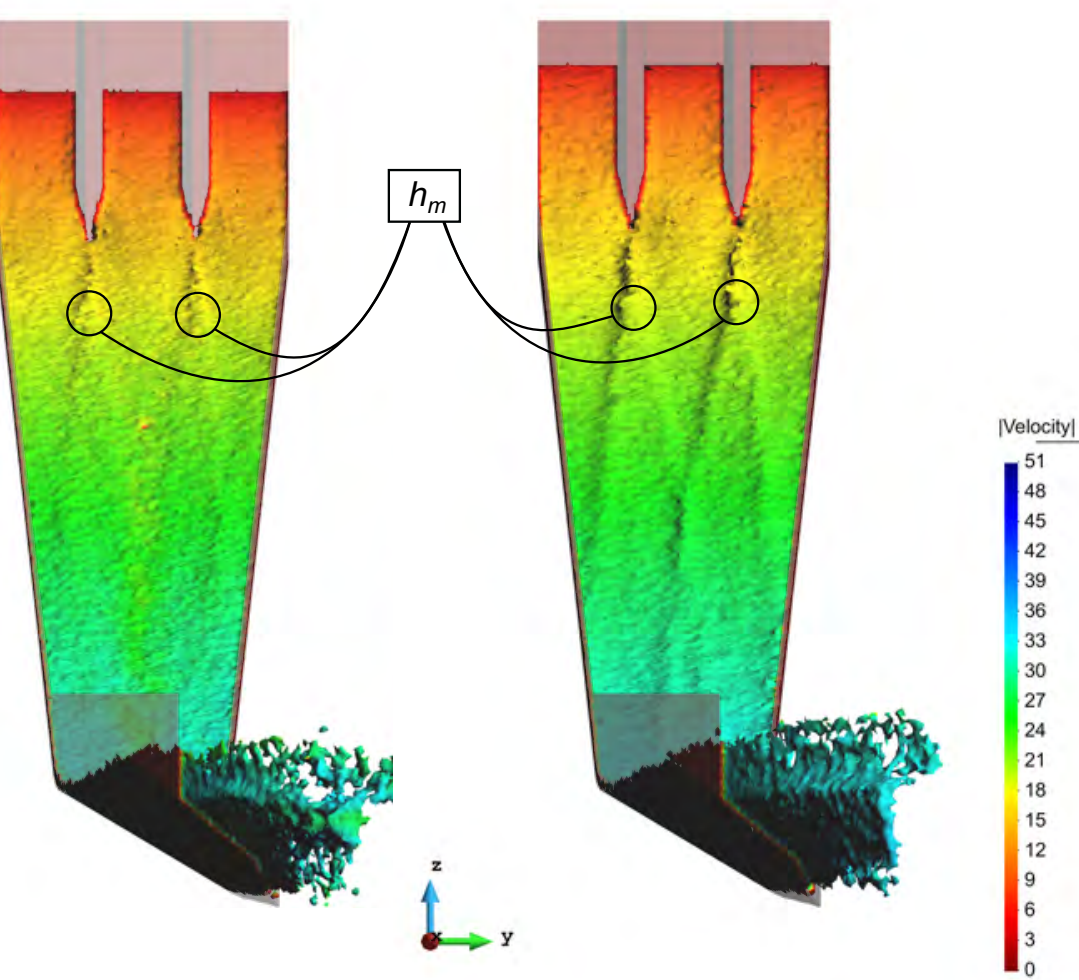

Fig. 17: Numerical results for $160 \mathrm{~m}^{3} / \mathrm{s}$ (left) and $450 \mathrm{~m}^{3} / \mathrm{s}$ (right). The location of the flow depth maxima is highlighted.

elliptical pier ends with equivalent dimensions (Figure 19). Table 6 shows the results.

\section{Discussion}

The results obtained in the test cases fit qualitatively with what was observed in the experiments. Similarly, the key magnitudes (maximum flow depth and its location) have been adequately reproduced with PFEM. Comparison with 

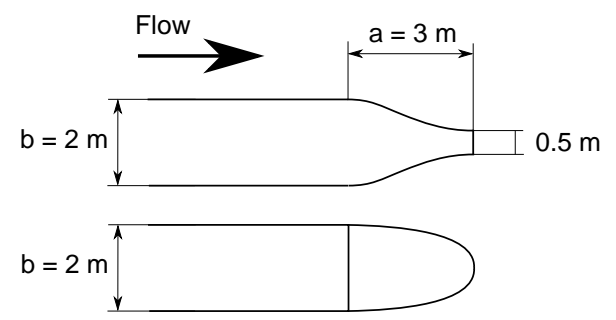

Fig. 19: Geometry of the piers in Case Study \#2 (top) and that for the equivalent semi-elliptical and rectangular (bottom).

Table 6: Comparison of experimental and empirical results for the flow depth maximum behind piers with $b=2 m$ and $i=0.469$

\begin{tabular}{llllll}
\hline & & & Rectangular & Case study \#2 & Elliptical \\
& & & Eq $15 ; a=0 m$ & Numerical & Eq $15 ; a=3 m$ \\
$\mathrm{Q}\left(\mathrm{m}^{3} / \mathrm{s}\right)$ & $h_{0}(m)$ & $h_{0} / b$ & $h_{m} / h_{0}$ & $h_{m} / h_{0}$ & $h_{m} / h_{0}$ \\
160 & 1.05 & 0.48 & 1.024 & 0.704 & 0.1 \\
450 & 2.3 & 1.04 & 1.52 & 0.786 & 0.52 \\
\hline
\end{tabular}


experimental cases should be analysed with caution as the information available from the tests is limited. It has already been mentioned that in some cases it is not easy to determine precisely the maximum flow depth, since either a relatively large area with similar depth is observed (Figure 4), or strong irregularities appear on the free surface (Figure 6).

In the cases analysed corresponding to contractions in rectangular channels, the angle formed by the shock wave front is slightly lower than that obtained from Equation 8. This causes the maximum depth to shift downstream in the numerical model, relative to the empirical estimate. Although in some cases it is not easy to determine the exact position of these maxima, this effect is clearly observed in the Figure 8.

The aforementioned shockwave front in contractions presents a slight curvature which has also been observed by Reinauer in laboratory [21].

The results of the Case Study \#1 offer a good approximation to the flow pattern observed in the laboratory. The quantitative comparison is limited because the experimental data correspond to some old tests for which some details are unknown such as the uncertainty in the depth measurements. Therefore, we consider that the most valuable result is the visual comparison with representative images (Figures 13 and 14). However, the numericalexperimental comparison of the flow depth along the right chute wall (Figure 16) gives similar results to those obtained for test cases: The numerical model offers a good approximation to the maximum depth, which are displaced downstream with respect to the experimental values. In this same Case Study \#1 
it is important to emphasize that the angle of the shockwave front fits with the theoretical estimate (Figure 15).

The Case Study \#2 has major limitations regarding quantitative evaluation of the results, as there are no experimental data to compare with. Furthermore, the geometry at the end of the piers does not correspond to those for which empirical formulas are available (rectangular, semi-elliptical). In spite of that, the results fit qualitatively with the observed behaviour, and the local depth maxima due to the roostertails behind the piles are observed, with magnitudes within the expected ranges taking into account the specific geometry.

\section{Summary and conclusions}

The possibilities of PFEM to model shock waves in hydraulic structures with supercritical regime have been shown in different configurations: laboratory tests corresponding to curves and convergence have been reproduced, and subsequently 2 spillways of real dams featuring different geometries have been modelled. The results show that PFEM is a useful tool for numerical modelling of this type of phenomena, offering a good approximation with an assumable computational cost, particularly for the first shock wave.

The main limitation of the presented approach is the lack of consideration of the interaction with air, which causes increase in flow depth in a supercritical regime [4]. In previous works on hydraulic assessment of bottom outlets, interaction between water and air was considered and meaningful results were obtained [28], [20]. However, consideration of air entrainment in spillways im- 
plies using a large domain of analysis to model the surrounding air, together with a dense mesh in the interface between both fluids, to account for their interaction. This results in excessive computational cost with the current version of the code. The authors are currently working in optimizing this PFEM implementation to consider aeration. The mentioned works on bottom outlets and other precedents [12] are the basis of the new developments.

The same problem arises in many laboratory tests, where aeration cannot be adequately modelled due to scale effects [11]. In spite of that, laboratory tests have been extensively used in the design and evaluation of dam spillways. Therefore, the model presented can be useful as a complement to experimental tests. The application of PFEM in these settings could reduce costs, enhance the analysis by considering flow patterns and stream lines and, above all, enable a wider spectrum of possible solutions to be considered, thanks to the greater flexibility of numerical models for the generation of geometries that can be laborious to build in the laboratory.

Acknowledgements The research was supported by the Spanish Ministry of Economy and Competitiveness (Ministerio de Economía y Competitividad, MINECO) through the project CALA (RTC-2016-4581-5).

\section{Conflict of interest}

On behalf of all authors, the corresponding author states that there is no conflict of interest. 


\section{References}

1. Aubry, R., Idelsohn, S., Oñate, E.: Particle finite element method in fluid-mechanics including thermal convection-diffusion. Computers \& Structures 83(17-18), 1459-1475 $(2005)$

2. Bayon, A., Valero, D., García-Bartual, R., López-Jiménez, P.A., et al.: Performance assessment of openfoam and flow-3d in the numerical modeling of a low reynolds number hydraulic jump. Environmental modelling \& software 80, 322-335 (2016)

3. Carbonell, J.M., Oñate, E., Suárez, B.: Modeling of ground excavation with the particle finite-element method. Journal of Engineering Mechanics 136(4), 455-463 (2009)

4. Chanson, H.: Stepped spillway flows and air entrainment. Canadian journal of civil engineering 20(3), 422-435 (1993)

5. Chow, V.: Open-Channel Hydraulics. McGraw-Hill, USA (1959)

6. Edelsbrunner, H., Mücke, E.P.: Three-dimensional alpha shapes. ACM Transactions on Graphics 13(1), 43-72 (1994)

7. Idelsohn, S., Mier-Torrecilla, M., Oñate, E.: Multi-fluid flows with the particle finite element method. Computer Methods in Applied Mechanics and Engineering 198(33), $2750-2767$ (2009)

8. Idelsohn, S., Oñate, E., Del Pin, F.: A lagrangian meshless finite element method applied to fluid-structure interaction problems. Computers and Structures 81(8), 655-671 (2003)

9. Idelsohn, S., Oñate, E., Pin, F.D.: The particle finite element method: a powerful tool to solve incompressible flows with free-surfaces and breaking waves. International Journal for Numerical Methods in Engineering 61(7), 964-989 (2004)

10. Larese, A., Rossi, R., Oñate, E., Idelsohn, S.R.: Validation of the particle finite element method (PFEM) for simulation of free surface flows. Engineering Computations 25(4), $385-425(2008)$

11. Lian, J., Qi, C., Liu, F., Gou, W., Pan, S., Ouyang, Q.: Air entrainment and air demand in the spillway tunnel at the jinping-i dam. Applied Sciences 7(9), 930 (2017) 
12. Nadukandi, P., Servan-Camas, B., Becker, P.A., Garcia-Espinosa, J.: Seakeeping with the semi-lagrangian particle finite element method. Computational Particle Mechanics 4(3), 321-329 (2017)

13. Oñate, E., Idelsohn, S.R., Celigueta, M.A., Rossi, R.: Advances in the particle finite element method for the analysis of fluid-multibody interaction and bed erosion in free surface flows. Computer Methods in Applied Mechanics and Engineering 197(19), 1777-1800 (2008)

14. Oñate, E., Rossi, R., Idelsohn, S.R., Butler, K.M.: Melting and spread of polymers in fire with the particle finite element method. International Journal for Numerical Methods in Engineering 81(8), 1046-1072 (2010)

15. Oñate, E., Celigueta, M.A., Idelsohn, S.R.: Modeling bed erosion in free surface flows by the particle finite element method. Acta Geotechnica 1(4), 237-252 (2006)

16. Oñate, E., Celigueta, M.A., Idelsohn, S.R., Salazar, F., Suárez, B.: Possibilities of the particle finite element method for fluid-soil-structure interaction problems. Computational Mechanics 48(3), 307-318 (2011)

17. Oñate, E., Franci, A., Carbonell, J.M.: Lagrangian formulation for finite element analysis of quasi-incompressible fluids with reduced mass losses. International Journal for Numerical Methods in Fluids 74(10), 699-731 (2014)

18. Oñate, E., Franci, A., Carbonell, J.M.: A particle finite element method for analysis of industrial forming processes. Computational Mechanics 54(1), 85-107 (2014)

19. Oñate, E., Idelsohn, S.: The particle finite element method. an overview. International Journal of Computational Methods 1, 267-307 (2004)

20. Pozo, D., Salazar, F., Toledo, M.: Modeling the hydraulic performance of the aeration system in dam bottom outlets using the particle finite element method. Revista Internacional de Métodos Numéricos para Cálculo y Diseño en Ingeniería 30(1), 51-59 (2014). [in Spanish]

21. Reinauer, R.: Kanalkontraktionen bei schiessendem abfluss und stosswellenreduktion mit diffraktoren. Ph.D. thesis, ETH Zurich (1995)

22. Reinauer, R., Hager, W.H.: Supercritical flow behind chute piers. Journal of Hydraulic Engineering 120(11), 1292-1308 (1994) 
23. Reinauer, R., Hager, W.H.: Supercritical bend flow. Journal of hydraulic engineering 123(3), 208-218 (1997)

24. Reinauer, R., Hager, W.H.: Supercritical flow in chute contraction. Journal of hydraulic Engineering 124(1), 55-64 (1998)

25. Salazar, F., Irazábal, J., Larese, A., Oñate, E.: Numerical modelling of landslidegenerated waves with the particle finite element method (PFEM) and a non-Newtonian flow model. International Journal for Numerical and Analytical Methods in Geomechanics (2015)

26. Salazar, F., Morán, R., Rossi, R., Oñate, E.: Analysis of the discharge capacity of radialgated spillways using cfd and ann-oliana dam case study. Journal of Hydraulic Research 51(3), 244-252 (2013)

27. Salazar, F., Oñate, E., Morán, R.: Numerical modelling of landslides in reservoirs via the particle finite element method (PFEM). Revista Internacional de Métodos Numéricos para Cálculo y Diseño en Ingeniería 28(2), 112-123 (2012). [in Spanish]

28. Salazar, F., San-Mauro, J., Celigueta, M.Á., Oñate, E.: Air demand estimation in bottom outlets with the particle finite element method. Computational Particle Mechanics 4(3), 345-356 (2017). DOI 10.1007/s40571-016-0117-4. URL https://doi.org/10.1007/s40571-016-0117-4

29. Saunders, K., Prakash, M., Cleary, P.W., Cordell, M.: Application of smoothed particle hydrodynamics for modelling gated spillway flows. Applied Mathematical Modelling 38(17-18), 4308-4322 (2014)

30. Teng, P., Yang, J.: Modeling and prototype testing of flows over flip-bucket aerators. Journal of Hydraulic Engineering 144(12), 04018,069 (2018)

31. USBR: El vado dam - service spillway modification - physical model study. Tech. Rep. HL-2017-02 (2017)

32. Valero, D., Bung, D.B., Crookston, B.M.: Energy dissipation of a type iii basin under design and adverse conditions for stepped and smooth spillways. Journal of Hydraulic Engineering 144(7), 04018,036 (2018) 
33. Valiani, A., Caleffi, V.: Brief analysis of shallow water equations suitability to numerically simulate supercritical flow in sharp bends. Journal of Hydraulic Engineering 131(10), 912-916 (2005)

34. Vischer, D., Hager, W.H., Cischer, D.: Dam hydraulics, vol. 2. Wiley Chichester, UK (1998)

35. Xue, H., Diao, M., Ma, Q., Sun, H.: Hydraulic characteristics and reduction measure for rooster tails behind spillway piers. Arabian Journal for Science and Engineering 43(10), 5597-5604 (2018)

36. Yang, J., Andreasson, P., Teng, P., Xie, Q.: The past and present of discharge capacity modeling for spillways — a swedish perspective. Fluids 4(1), 10 (2019)

37. Zienkiewicz, O., Ortiz, P.: A split-characteristic based finite element model for the shallow water equations. International Journal for Numerical Methods in Fluids 20(8-9), $1061-1080(1995)$

38. Zienkiewicz, O.C., Taylor, R.L.: The finite element method for solid and structural mechanics. Butterworth-Heinemann (2005) 